LETTER TO THE EDITOR

\title{
Charge and Density Fluctuations Lock Horns : Ionic Criticality with Power-Law Forces
}

\author{
Jean-Noël Aqua and Michael E. Fisher \\ Institute for Physical Science and Technology, University of Maryland, College \\ Park, Maryland 20742, USA \\ E-mail: jnaqua@glue.umd.edu, xpectnil@ipst.umd.edu
}

\begin{abstract}
How do charge and density fluctuations compete in ionic fluids near gas-liquid criticality when quantum mechanical effects play a role? To gain some insight, long-range $\Phi_{ \pm \pm}^{\mathcal{L}} / r^{d+\sigma}$ interactions (with $\sigma>0$ ), that encompass van der Waals forces (when $\sigma=d=3$ ), have been incorporated in exactly soluble, $d$ dimensional 1:1 ionic spherical models with charges $\pm q_{0}$ and hard-core repulsions. In accord with previous work, when $d>\min \{\sigma, 2\}$ (and $q_{0}$ is not too large), the Coulomb interactions do not alter the $\left(q_{0}=0\right)$ critical universality class that is characterized by density correlations at criticality decaying as $1 / r^{d-2+\eta}$ with $\eta=\max \{0,2-\sigma\}$. But screening is now algebraic, the charge-charge correlations decaying, in general, only as $1 / r^{d+\sigma+4}$; thus $\sigma=3$ faithfully mimics known noncritical $d=3$ quantal effects. But in the absence of full $(+,-)$ ion symmetry, density and charge fluctuations mix via a transparent mechanism: then the screening at criticality is weaker by a factor $r^{4-2 \eta}$. Furthermore, the otherwise valid Stillinger-Lovett sum rule fails at criticality whenever $\eta=0$ (as, e.g., when $\sigma>2$ ) although it remains valid if $\eta>0$ (as for $\sigma<2$ or in real $d \leq 3$ Ising-type systems).
\end{abstract}

An ionic fluid, such as an electrolyte or a plasma, is characterized in thermal equilibrium by the screening of the long-range Coulomb interaction potential, $z_{\tau} z_{v} q_{0}^{2} / r^{d-2}$ (say, in $d>2$ dimensions) between ions of charges $z_{\tau} q_{0}$ and $z_{v} q_{0}$. Following Debye-Hückel theory [1] one expects the charge-charge correlation function, $\ddagger$ $G_{Z Z}(\mathbf{r})$, to decay exponentially as $e^{-r / \xi_{Z, \infty}}$ where, as the overall ion density $\rho$ becomes small, the screening length $\xi_{z, \infty}(T, \rho)$ should approach the Debye length $\xi_{D} \equiv 1 / \kappa_{D} \propto \sqrt{T / q_{0}^{2} \rho}$. More generally, the screening of an external charge in a conductor should be characterized by the Stillinger-Lovett sum rule [1, 3] which does not require exponential screening [4]; this states that when $\mathbf{k} \rightarrow \mathbf{0}$, the charge structure factor, essentially the Fourier transform $\ddagger$ of $G_{Z Z}(\mathbf{r})$, behaves as

$$
S_{Z Z}(\mathbf{k})=0+\xi_{z, 1}^{2} k^{2}-\xi_{z, 2}^{4} k^{4}-\xi_{z, \varsigma}^{\psi} k^{\psi}+\ldots \quad \text { with } \quad \xi_{z, 1}=\xi_{D} .
$$

Note also that the vanishing leading term simply reflects the requirement of electroneutrality; if the screening is exponential only the further powers $k^{2 l}$ with $l=1,2, \ldots$ can arise; but, in general, which specific, nonanalytic powers appear and with what amplitudes is a matter of prime interest.

$\ddagger$ Our notations, which are fairly standard [1] are set out explicitly in [2 which will be denoted AF. 
The exponential screening of charge has been proven rigorously at low densities for classical systems [5]; but sufficiently strong short-distance repulsions between oppositely charged ions are essential while any further non-Coulomb interaction potentials, say $\varphi_{\tau v}(\mathbf{r})$ between ions of species $\tau$ and $v$, must be of short-range, decaying, e.g., exponentially fast when $r \rightarrow \infty$. But then, in allowing for quantum mechanics, one should first recognize that real polarizable ions also interact via fluctuating induced dipole-dipole or van der Waals forces that fall off only algebraically. Specifically, if for generality we consider integrable long-range potentials that decay as $\Phi_{\tau v}^{\mathcal{L}} / r^{d+\sigma}$ (so requiring $\sigma>0$ ) [6], van der Waals forces may be described by $d=\sigma=3$. Then one may ask what will be the consequences of such long-range power-law forces for exponential screening and for the expansion (1).

However, even in a point-ion model of a plasma, the quantum-mechanical fluctuations of position lead to effective particle-particle potentials, as manifest in the corresponding correlation functions, that decrease as $1 / r^{6}$ (for $d=3$ ) [7] [8], just as for polarizable ions (or neutral species). Furthermore, it is known that in quantum plasmas at low density, the screening of charge is no longer exponential: indeed, $G_{Z Z}(\mathbf{r})$ has recently been shown to decay as $1 / r^{10}$ [7, 8].

On approach to gas-liquid or liquid-liquid criticality, in an ionic system, the situation is further complicated because the density fluctuations become divergent and it is natural to ask if this should not seriously affect charge screening, the StillingerLovett rule, etc. To be more explicit, in a short-range, nonionic system, the densitydensity correlation function $G_{N N}(\mathbf{r})$ decays as $e^{-r / \xi_{N}, \infty}$ where, on the critical isochore $\rho=\rho_{c}$, the density correlation length $\xi_{N, \infty}(T, \rho)$ diverges as $1 / t^{\nu}$ with $t \equiv\left(T-T_{c}\right) / T_{c}$ and $\nu \geq \frac{1}{2}$; but at criticality, one has

$$
G_{N N}^{c}(\mathbf{r}) \equiv D_{N N}^{c} / r^{d-2+\eta} \quad \text { when } r \rightarrow \infty
$$

with $D_{N N}^{c}$ finite and $\eta \geq 0$. Might not the divergence of $\xi_{N, \infty}$ couple in some way to the screening length $\xi_{z, \infty}$ in a corresponding $\left(\Phi_{\tau v}^{\mathcal{L}} \equiv 0\right)$ classical plasma and cause it to diverge at criticality? Likewise, might not the slow decay of $G_{N N}(\mathbf{r})$ at criticality change the $1 / r^{10}$ quantal decay of $G_{Z Z}(\mathbf{r})$ that has been established at low densities?

Of course, the values of the critical exponents $\nu$ and $\eta$ depend on the critical universality class, and the influence of Coulomb couplings on criticality has been an important experimental and theoretical question ever since seemingly convincing observations on certain electrolytes suggested that ionic criticality might realize a new or different type of critical behavior [9-11]. While the experimental issues for electrolytes may now be regarded as largely settled - in favor of no change in critical character - the theoretical situation remains open. And neither experimentally nor theoretically has a clear picture of the charge correlations near criticality yet emerged.

One question of particular relevance [11] concerns the role of ion-symmetry. Thus, the simplest theoretical description of a 1:1 electrolyte is afforded by the so-called restricted primitive model or RPM, in which equisized hard spheres carry charges $+q_{0}$ and $-q_{0}$. The RPM is precisely ion symmetric so that it is plausible that charge and density fluctuations will remain effectively independent even at criticality. Indeed, careful simulations [12, 13, have recently established that criticality in the $d=3 \mathrm{RPM}$ still exhibits Ising behavior as do simple, nonionic fluids. But in reality, + and - ions are never identical so that ion nonsymmetric models are of especial interest. While simulations of 1:1, 2:1 and 3:1 hard sphere models with unequal diameters have been undertaken [14, their critical behavior has not, as yet, been resolved. 
To address some of these problems, we have recently analyzed [2] a class of exactly soluble $d$-dimensional multicomponent spherical models in which particles of different species $\tau, v, \ldots$ reside on distinct but equivalent interlaced sublattices with nearest neighbor spacing $a$. Then, in a 1:1 'lattice electrolyte,' nearby ions of species + and - with charges $\pm q_{0}$ avoid collapse because opposite charges are never closer than the minimal, intersublattice distance, say $a_{0} \lesssim a\left[2\right.$. In addition to the $\pm q_{0}^{2} / r^{d-2}$ Coulomb potentials, short-range attractive potentials $\varphi_{ \pm \pm}^{0}(\mathbf{r})$ of magnitude, say, $k_{B} T_{0}$ are then introduced; on setting $q_{0}=0$, these suffice to yield standard spherical model critical behavior, with $\eta=0$ etc., at $T_{c}\left(q_{0}=0\right) \simeq T_{0}[2]$.

Two main conclusions emerge from AF [2. First, the character of criticality remains unchanged when the charges are switched on (provided $q_{0}$ is not too large). Second, ion symmetry plays a crucial role: thus in symmetric models, the charge screening length $\xi_{z, \infty}(T, \rho)$ remains of order $\xi_{D}$ even at $\left(T_{c}, \rho_{c}\right)$ although it gains singular corrections when $t \rightarrow 0$; but, on the contrary, for asymmetric fluids, $\xi_{z, \infty}$ diverges on approach to criticality, precisely matching the density correlation length $\xi_{N, \infty} \sim 1 / t^{\nu}$. Furthermore, the Stillinger-Lovett rule is then violated at criticality. Here we extend $\mathbf{A F}$ by incorporating additional, long-range $\Phi_{ \pm \pm}^{\mathcal{L}} / r^{d+\sigma}$ ionion potentials in order to cast some light on the further role that quantum mechanical fluctuations might play. (To avoid technical complications we suppose $\sigma \neq 2,4, \cdots$.) As we report, the findings prove instructive.

The necessary analysis follows closely the lines set out in AF: accordingly we focus on the principal results sketching only the basic technical points. (Further and fuller details will be presented elsewhere [15].) Thus Lagrange multipliers $\left(\lambda_{+}, \lambda_{-}\right) \equiv \boldsymbol{\lambda}(T, \rho)$ are introduced to satisfy the spherical model constraints $\left\langle s_{\tau}^{2}\right\rangle=1$ for $\tau=+,-$, where the $s_{\tau}(\mathbf{R})$ are the usual unbounded scalar spin variables [16-19]. The free energy then follows from the eigenvalues, $\Lambda_{N}(\mathbf{k} ; \boldsymbol{\lambda})$ and $\Lambda_{Z}(\mathbf{k} ; \boldsymbol{\lambda})$, of the interaction matrix $\boldsymbol{\Lambda}$ with elements of the form $\Lambda_{\tau v}=\left[\lambda_{\tau} \delta_{\tau v}-\tilde{\varphi}_{\tau v}(\mathbf{k})\right]$, where the $\tilde{\varphi}_{\tau v}$ are proportional to the Fourier transforms of the total interaction potentials $\varphi_{\tau v}(\mathbf{r})$.

Now, as in $\mathbf{A F}$, the crucial result is that the $q_{0}^{2} / k^{2}$ Coulomb divergence cancels out identically from the first eigenvalue which, at small wave numbers $k \equiv|\mathbf{k}|$, then behaves as

$$
\Lambda_{N}(\mathbf{k} ; \boldsymbol{\lambda})=\frac{1}{2} k_{B} T_{0}\left[\dot{\lambda}(T, \rho)+\left(R_{N} k\right)^{2}+\left(R_{N}^{\mathcal{L}} k\right)^{\sigma}+\ldots\right],
$$

where $\dot{\lambda}\left(\equiv \lambda / j_{0}\right.$ in the notation of $\left.\mathbf{A F}\right)$ is found to vanish on the critical isochore near criticality like $t^{\gamma}$, while the finite, nonzero length $R_{N}\left(\boldsymbol{\lambda} ; q_{0}\right)$ measures the range of the short range forces [see $\mathbf{A F}$ (25)]. The relative contribution of the long range interactions to the density variation is embodied in the effective range $R_{N}^{\mathcal{L}}$ [15]. From this form for $\Lambda_{N}$ one finds (for $q_{0}$ not too large) that the critical behavior is always of spherical model form with exponents $\beta=\frac{1}{2}$ and

$$
\eta(\sigma)=\max \{0,2-\sigma\}, \quad \gamma=1-\alpha=(2-\eta) \nu=\frac{2-\eta(\sigma)}{d-2+\eta(\sigma)},(4)
$$

[6, 16, 19] while $d>\min \{\sigma, 2\}$ is needed for $T_{c}>0$ [and we suppose $\left(R_{N}^{\mathcal{L}}\right)^{\sigma}>0$ when $\sigma<2]$. For $\sigma>2$ these leading exponents are, as well known, independent of $\sigma$ and the same as for short-range forces; but see also [20]. However, new correction terms varying as $t^{\theta_{\sigma}}$ with $\theta_{\sigma}=|2-\sigma| \nu(\sigma)$ will dominate in all properties when $\sigma$ is close to 2 .

The long range effects of the Coulomb forces appear only in the second eigenvalue which, for small $k$, varies as

$$
\Lambda_{z}(\mathbf{k} ; \boldsymbol{\lambda})=\frac{S_{d}}{4 a^{d}} \frac{q_{0}^{2}}{k^{2}}\left[1+\left(R_{z} k\right)^{2}+\left(R_{z}^{\mathcal{L}} k\right)^{2+\sigma}+\ldots\right],
$$


with $S_{d}=2 \pi^{d / 2} / \Gamma(d / 2)$. The net contribution of the long range forces is now represented by $R_{z}^{\mathcal{L}}$ while $R_{Z}(\boldsymbol{\lambda} ; \mathrm{k} / k)$ is of order $a / \sqrt{\mathcal{I}_{0}} \sim a / q_{0}$ where the ionicity

$$
\mathcal{I}_{0}=q_{0}^{2} / a^{d-2} k_{B} T_{0},
$$

measures the overall strength of the Coulomb interactions near criticality. [It should be noted that the ellipses in (3) and (5) include both further singular terms and analytic terms of order $k^{4}, k^{6}, \cdots$.]

Finally, we may express the charge and density structure factors in the transparent form

$$
\begin{aligned}
\frac{S_{N N}(\mathbf{k})}{k_{B} T / 4 \rho a^{d}}=\frac{1-\delta_{\varphi} B(\mathbf{k})}{\Lambda_{N}(\mathbf{k} ; \boldsymbol{\lambda})}+\frac{\delta_{\varphi} B(\mathbf{k})}{\Lambda_{Z}(\mathbf{k} ; \boldsymbol{\lambda})}, \\
\frac{S_{Z Z}(\mathbf{k})}{k_{B} T / 4 \rho a^{d}}=\frac{\delta_{\varphi} B(\mathbf{k})}{\Lambda_{N}(\mathbf{k} ; \boldsymbol{\lambda})}+\frac{1-\delta_{\varphi} B(\mathbf{k})}{\Lambda_{Z}(\mathbf{k} ; \boldsymbol{\lambda})},
\end{aligned}
$$

where the basic symmetry parameter $\delta_{\varphi}\left(=\lambda^{\dagger} / j_{0}\right.$ in the notation of $\left.\mathbf{A F}\right)$ vanishes linearly with the deviation of the potentials $\varphi_{\tau v}$ from precise (or effective) ion symmetry. Clearly the density and charge fluctuations in ion symmetric models are completely uncoupled (at least in quadratic order): the critical density fluctuations are thus driven solely by $\Lambda_{N}(\mathbf{k})$ while charge screening is entirely controlled by $\Lambda_{Z}(\mathbf{k})$. But, charge and density fluctuations mix as soon as ion symmetry is lost: to what degree is determined by

$$
B(\mathbf{k})=4 k^{4} a^{4}\left[\delta_{\varphi}+\left(R_{\varphi}^{\mathcal{L}} k\right)^{\sigma}+\ldots\right] / S_{d}^{2} \mathcal{I}_{0}^{2},
$$

where $R_{\varphi}^{\mathcal{L}}$ measures the strength of the asymmetric parts of the long range forces (and hence vanishes with $\delta_{\varphi}$ ). A decomposition similar to (7) holds for $S_{N Z}$ : see $\mathbf{A F}$.

The factor $k^{4}$ in (8) implies that the "intrinsic" charge fluctuations contribute only weakly to $S_{N N}$. Consequently, except $a t\left(T_{c}, \rho_{c}\right)$, one has, neglecting analytic background terms of order $k^{4}$,

$$
S_{N N}(\mathbf{k}) \propto \frac{\chi_{T}}{1+\xi_{N, 1}^{2} k^{2}+\xi_{N, \sigma}^{\sigma} k^{\sigma}+\ldots}+\mathcal{O}\left(\delta_{\varphi} k^{4+\sigma}\right),
$$

where $\chi_{T} \sim 1 / t^{\gamma}$ is the isothermal compressibility, while the new length scales are

$$
\xi_{N, 1}(T, \rho)=R_{N} / \dot{\lambda}^{1 / 2} \quad \text { and } \quad \xi_{N, \sigma}(T, \rho)=R_{N}^{\mathcal{L}} / \dot{\lambda}^{1 / \sigma} .
$$

Since $\dot{\lambda}$ vanishes like $t^{\gamma}$ on approaching criticality, the density correlation length may be identified as $\xi_{N}(T, \rho)=\max \left\{\xi_{N, 1}, \xi_{N, \sigma}\right\}$ in full accord with the exponent values (44).

Likewise, the critical point decay (2) with $\eta=\max \{0,2-\sigma\}$ is readily verified. Away from criticality, however, matters are somewhat more subtle since, in general, no correlation function, $G_{\tau v}(\mathbf{r})$, can decay faster than the associated $1 / r^{d+\sigma}$ power-law potentials [21. Indeed, the first nonanalyticity in $S_{N N}(\mathbf{k})$ yields the large- $r$ behavior [22] whence we find, for fixed $(T, \rho) \neq\left(T_{c}, \rho_{c}\right)$,

$$
\begin{aligned}
G_{N N}(\mathbf{r} ; T, \rho) & \approx \frac{D_{N, \sigma}}{r^{d-2+\eta}}\left(\frac{\xi_{N}}{r}\right)^{2 \sigma} \sim \frac{1}{r^{d+\sigma}} \quad \text { when } \sigma<2, \\
& \approx \frac{D_{N, \sigma}}{r^{d-2}}\left(\frac{\xi_{N}}{r}\right)^{2}\left(\frac{\xi_{N, \sigma}}{r}\right)^{\sigma} \sim \frac{1}{r^{d+\sigma}} \quad \text { when } \sigma>2 .
\end{aligned}
$$

The appearance of the factor $\left(\xi_{N, \sigma} / r\right)$ reflects the correction-to-scaling exponent $\theta_{\sigma}=|2-\sigma| \nu(\sigma)$ identified above.

Now we may study the charge correlations and ask, to start with, about the noncritical behavior. When ion symmetry pertains, i.e. $\delta_{\varphi}=0$, the decomposition 
(7) shows that $S_{z z}$ depends only on $\Lambda_{z}$; then (5) leads directly to the expansion (11) with, furthermore, full confirmation of the Stillinger-Lovett (SL) relation 2, 17, (since $\kappa_{D}^{2}=S_{d} \rho q_{0}^{2} / k_{B} T$ ). But, by virtue of the factor $k^{4}$ in (8), this remains true even when ion symmetry is absent. In addition, the coefficient of $k^{4}$ in (11) is given by

$$
\xi_{z, 2}^{4}=\xi_{D}^{2}\left[R_{z}^{2}-b_{0} \delta_{\varphi}^{2} \xi_{N, \sigma}^{\sigma}(T, \rho)\right]
$$

with $b_{0}=\mathcal{O}\left(a^{2-\sigma}\right)$. Evidently, this moment of $G_{Z Z}$ is unconstrained and if $\delta_{\varphi} \neq 0$ it will change sign when, driven by the coupling to the density fluctuations embodied in (7), $\xi_{N, \sigma}^{\sigma}$ diverges like $1 / t^{\gamma}$ as $T \rightarrow T_{c}$ on the critical isochore: see (10).

Lastly, the leading nonanalytic term in $S_{z z}(\mathbf{k})$ is given, in (1), by $\psi=4+\sigma$ while its amplitude is

$$
\xi_{z, \varsigma}^{4+\sigma}=\xi_{D}^{2}\left[\left(R_{z}^{\mathcal{L}}\right)^{2+\sigma}-b_{1} \delta_{\varphi}\left(R_{\varphi}^{\mathcal{L}} \xi_{N, \sigma}\right)^{\sigma}+b_{0} \delta_{\varphi}^{2} \xi_{N, \sigma}^{2 \sigma}\right]
$$

where $b_{1}=\mathcal{O}\left(a^{2-\sigma}\right)$. Clearly, this $k^{4+\sigma}$ term is present whether or not ion symmetry pertains; but if $\delta_{\varphi} \neq 0$ it may change sign and its magnitude will diverge, like $1 / t^{2 \gamma}$, when $(T, \rho) \rightarrow\left(T_{c}, \rho_{c}\right)$. More strikingly, however, the presence of this term ensures the destruction of exponential screening; rather one finds [22]

$$
G_{Z Z}(\mathbf{r} ; T, \rho) \approx \frac{D_{Z, \sigma}}{r^{d}}\left(\frac{\xi_{Z, \varsigma}}{r}\right)^{4+\sigma} \sim \frac{1}{r^{d+\sigma+4}} .
$$

Thus near to or far from criticality, long range forces always undermine the standard picture of Debye screening. Nevertheless, algebraic screening remains in the charge correlations. Indeed, we may say that the $1 / r^{d-2}$ Coulomb potential is screened by a factor $1 / r^{6+\sigma}$ or, equivalently, that the long range $1 / r^{d+\sigma}$ potential is screened owing to the requirements of "local electroneutrality"- by the factor $1 / r^{4}$. This is a central result of our analysis and it is gratifying that on setting $\sigma=d=3$ it reproduces the $1 / r^{10}$ screening previously found in a fully quantum mechanical analysis of pointcharge plasmas [7, 8].

In contrast to the loss of exponential screening, ion symmetry is paramount at criticality. Thus we see from (5) and (7) that all the results (12)-(14) remain uniformly valid when $(T, \rho) \rightarrow\left(T_{c}, \rho_{c}\right)$ provided $\delta_{\varphi}=0$, i.e., that ion symmetry is valid: one need only note that the amplitudes $R_{z}^{2}$ and $D_{z, \sigma}\left(\xi_{z, \varsigma}\right)^{4+\sigma}$ in (12) and (14) remain finite (and nonzero) at the critical point although the former will pick up a singular $t^{1-\alpha}$ correction (as in AF: see [15]).

On the other hand, for nonsymmetric fluids at criticality, the mixing of the charge and density fluctuations depends strongly on $\sigma$ or, more specifically, on $\eta(\sigma)$. Indeed, displaying only the leading singular terms, we find

$$
\begin{aligned}
& S_{Z Z}^{c}(\mathbf{k})=\xi_{D, c}^{2} k^{2}\left[1+\delta_{\varphi}^{2}\left(R_{>} k\right)^{2-\sigma}+\mathcal{O}\left(k^{2}\right)\right] \text { for } \eta=2-\sigma>0, \\
& =\xi_{D, c}^{2} k^{2}\left[\mathcal{E}_{c}-\delta_{\varphi}^{2}\left(R_{<} k\right)^{\sigma-2}+\mathcal{O}\left(k^{2}\right)\right] \text { for } \eta=0<\sigma-2,
\end{aligned}
$$

where $R_{>}^{2-\sigma}=\tilde{a}^{2}\left(R_{N}^{\mathcal{L}}\right)^{-\sigma}, \quad R_{<}^{\sigma-2}=\tilde{a}^{2}\left(R_{N}^{\mathcal{L}}\right)^{\sigma} / R_{N}^{4}, \quad \mathcal{E}_{c}=1+\delta_{\varphi}^{2} \tilde{a}^{2} / R_{N}^{2}>1$ with $\tilde{a}\left(q_{0}\right)=a \sqrt{2 / S_{d} \mathcal{I}_{0}}$ : see $\mathbf{A F}(29)$. One sees immediately that the Stillinger-Lovett sum rule remains valid whenever $\sigma<2$ or $\eta>0$. This contrasts with the results of $\mathbf{A F}$ where, in the absence of long-range forces, one has $\eta=0$ and the sum-rule is violated, precisely the situation that prevails here when $\sigma>2$; then the critical system may be regarded as an insulator or, at least, as an anomalous conductor! 
Table 1. Long-distance behavior of charge correlations $G_{Z Z}(\mathbf{r})$ at fixed $(T, \rho)$. The density correlation length $\xi_{N}$ diverges on the critical isochore $\rho=\rho_{c}$ as $1 / t^{\nu}$ when $t=\left(T-T_{c}\right) / T_{c} \rightarrow 0$. The density correlations decay as $1 / r^{d+\sigma}$ away from criticality, but at criticality, since $\eta=\max \{0,2-\sigma\}$, they decrease more slowly as $1 / r^{d-\sigma}$ when $\sigma<2$ and $1 / r^{d-2}$ when $\sigma>2$.

\begin{tabular}{cccc}
\hline$G_{Z Z}(\mathbf{r})$ & ion symmetric & \multicolumn{3}{c}{ nonsymmetric } \\
$\delta_{\varphi}=0$ & & $\sigma<2$ & $\sigma>2$ \\
\hline$(T, \rho)$ & & $\sim 1 / r^{d+4-\sigma}$ & $\sim 1 / r^{d+\sigma}$ \\
\hline$\left(T_{c}, \rho_{c}\right)$, & $\sim 1 / r^{d+\sigma+4}$ & $\sim\left(\xi_{N} / r\right)^{4-2 \eta} / r^{d+4-\sigma}$ & $\sim\left(\xi_{N} / r\right)^{4} / r^{d+\sigma}$ \\
$\neq\left(T_{c}, \rho_{c}\right)$, & $\sim 1 / r^{d+\sigma+4}$ & \\
\hline
\end{tabular}

From (15) we can now deduce the long-range behavior of the critical-point chargecharge correlation function, namely

$$
\begin{aligned}
G_{Z Z}^{c}(\mathbf{r}) & \approx \frac{D_{Z, \sigma}^{c} \delta_{\varphi}^{2} R_{>}^{2-\sigma}}{r^{d+4-\sigma}} \sim \frac{1}{r^{d-2+\eta}} \frac{1}{r^{4}} \quad \text { for } \quad \eta(\sigma)>0, \\
& \approx \frac{D_{Z, \sigma}^{c} \delta_{\varphi}^{2} R_{<}^{\sigma-2}}{r^{d+\sigma}} \sim \frac{1}{r^{d-2+\eta}} \frac{1}{r^{2+\sigma}} \quad \text { for } \quad \eta=0<\sigma-2 .
\end{aligned}
$$

As displayed, the results show that although $G_{Z Z}^{c}(\mathbf{r})$ is driven by the critical density fluctuations, with $G_{N N}^{c} \sim 1 / r^{d-2+\eta}$, the charge correlations are screened relatively more strongly when $\sigma>2$ than for $\sigma<2$, when $\eta>0$. In light of the failure of the SL rule in the former case rather than the latter, this is, perhaps, paradoxical. On the other hand, one might equally conclude that the long-range $1 / r^{d+\sigma}$ potentials are not screened, even algebraically, when $\sigma>2$ (with $\eta=0$ ) whereas for $\sigma<2$ the long-range forces are actually screened by factors $1 / r^{2 \eta}=4-2 \sigma$. From that perspective the validity of the SL rule when $\eta>0$ seems more natural [11].

Finally, similar conclusions can be drawn about the charge-density structure factor: see AF. Away from criticality we find

$$
S_{N Z}(\mathbf{k})=\delta_{\varphi} \dot{\lambda}^{-1} \xi_{D}^{2} k^{2}\left[1-\left(\tilde{\xi}_{N, \sigma} k\right)^{\sigma}+\ldots\right],
$$

where $\tilde{\xi}_{N, \sigma}^{\sigma}=\xi_{N, \sigma}^{\sigma}-\left(R_{\varphi}^{\mathcal{L}}\right)^{\sigma} / \delta_{\varphi}$ and one should recall from (10) that $\left|\xi_{N, \sigma}^{\sigma}(T, \rho)\right| \propto$ $1 / \dot{\lambda}(T, \rho)$ diverges like $1 / t^{\gamma}$ while, by (8), $R_{\varphi}^{\mathcal{L}}$ vanishes with $\delta_{\varphi}$. In real space the long-range decay can be written as

$$
G_{N Z}(\mathbf{r} ; T, \rho) \approx \frac{\delta_{\varphi} D_{N Z}}{r^{d+|\sigma-2|}}\left(\frac{\xi_{N}}{r}\right)^{4-2 \eta(\sigma)} \sim \frac{1}{r^{d+\sigma+2}},
$$

so that the cross-correlation function is evidently screened by the factor $1 / r^{2}$ relative to the long range potentials. Alternatively, one may say that the $1 / r^{d-2}$ Coulomb interaction is screened by a factor $1 / r^{4+\sigma}$. As regards the "van der Waals case" $d=\sigma=3$, we may mention that the charge density induced by an infinitesimal local external charge decays as $1 / r^{8}$ in a fully quantal point-charge plasma [7, 8].

At the critical point itself, one finds that $S_{N Z}^{c}(\mathbf{0})$ vanishes identically if $\eta>0$ $(\sigma<2)$ but that, as in the short-range case [2], $S_{N Z}^{c}(\mathbf{0})=\delta_{\varphi}\left(\xi_{D} / R_{N}\right)_{c}^{2} \neq 0$, whenever $\eta=0(\sigma>2)$. Moreover, when $r \rightarrow \infty$ one has $G_{N Z}^{c}(\mathbf{r}) \sim \delta_{\varphi} / r^{d+|\sigma-2|}$ so that the infection of the charge correlations by the density fluctuations again reduces the 
screening by a factor $r^{4-2 \eta}$ that is greatest when $\eta=0$ : Compare with the results for $G_{Z Z}(\mathbf{r})$ as displayed in Table 1 .

In conclusion, we have analyzed the interplay between long-range density fluctuations, Coulomb interactions, and power-law $1 / r^{d+\sigma}$ (e.g., van der Waals) interactions, away from, close to and at criticality in ionic fluids, on the basis of two-component, $d$-dimensional spherical models with hard-core interspecies repulsions [2]. Throughout the fluid phase, including low-densities, the power-law forces destroy the usual Debye exponential screening. However, algebraic charge-charge and density-charge screening is still present: explicitly, although the density-density correlation function $G_{N N}(\mathbf{r})$ decays no faster than the interaction potentials [21], i.e., as $1 / r^{d+\sigma}$, the charge-charge correlation function, $G_{Z Z}(\mathbf{r})$, decays at large distances as $D_{z}(T, \rho) / r^{d+\sigma+4}$. If one sets $\sigma=3$ to mimic the $1 / r^{6}$ particle-particle interactions that arise in $d=3$ dimensions from quantal fluctuations [7, 8], this result is, indeed, in accord with exact results for $G_{Z Z}(\mathbf{r})$ in a fully quantum-mechanical, point-charge plasma at low densities [7, 8].

In the critical region, the Coulomb interactions leave the universality class of the spherical models unchanged since, as in $\mathbf{A F}$, they still cancel out of the fluctuation factor that drives criticality. Nevertheless, radical changes arise in the chargecharge and charge-density fluctuations, whether the system is ion symmetric or, more realistically, nonsymmetric. The behavior is enforced by a general decomposition of the structure factors: see (7) and [2]. For ion symmetric fluids, the asymptotic amplitude $D_{z}(T, \rho)$ is always finite and the Stillinger-Lovett (SL) sum rule is satisfied even at criticality. For asymmetric systems near criticality, $D_{z}$ is driven by the density fluctuations and hence diverges as $1 / t^{2 \gamma}$ when $\rho=\rho_{c}$ while the SL sum rule remains valid. At criticality, however, the density correlations, decaying now as $1 / r^{d-2+\eta}$, weaken the charge screening still further, more strongly when $\eta=0(\sigma>2)$ than if $\eta>0(\sigma<2)$; see the summary in Table I. Finally, the SL sum rule, characteristic of conductors, is satisfied at criticality when $\eta>0$, but is violated when $\eta=0$, a result that may well have validity beyond the ionic spherical models studied here [11. A further challenge is to see how far similar results might be obtained for intrinsically quantal spherical models such as have been advanced in the past [18,23-25].

\section{Acknowledgments}

Support from the NSF (under grant CHE 03-01101) as well as assistance to J.-N.A. from the French Ministry of Foreign Affairs under the Lavoisier Fellowship program, is gratefully acknowledged.

\section{References}

[1] See, e.g., Hansen J-P and McDonald I R 1986 Theory of simple liquids (London: Academic Press)

[2] Aqua J-N and Fisher M E 2004 Phys. Rev. Lett. 92 135702, to be denoted AF

[3] Stillinger F H and Lovett R 1968 J. Chem. Phys. 483858

[4] Martin P-A 1988 Rev. Mod. Phys. 601075

[5] Brydges D C and Federbush P 1980 Commun. Math. Phys. 73197

[6] Fisher M E, Ma S K and Nickel B G 1972 Phys. Rev. Lett. 29917

[7] Alastuey A and Martin P-A 1989 Phys. Rev. A 406485

[8] Cornu F 1996 Phys. Rev. E 534595

[9] Weingärtner W and Schröer W 2001 Adv. Chem. Phys. 1161

[10] Fisher M E 1994 J. Stat. Phys. 751 
[11] Stell G 1995 J. Stat. Phys. 78197

[12] Luijten E, Fisher M E and Panagiotopoulos A Z 2002 Phys. Rev. Lett. 88185701

[13] Kim Y C and Fisher M E 2004 Phys. Rev. Lett. 92 in press

[14] Panagiotopoulos A Z and Fisher M E 2002 Phys. Rev. Lett. 88045701

[15] Aqua J-N and Fisher M E in preparation

[16] Joyce G S 1972 in Phase transitions and Critical Phenomena vol 2 Eds Domb C and Green M S (New-York: Academic) p 375

[17] Smith E R 1988 J. Stat. Phys. 50813

[18] Brankov J G, Danchev D M and Tonchev N S 2000 Theroy of Critical Phenomena in Finite-Size Systems (Singapore: World Scientific) Chap 3

[19] Fisher M E 2004 in Current Topics in Physics Eds Barrio R A and Kaski K K (U.K.: Imperial College Press)

[20] Dantchev D 2001 Eur. Phys. J. B 23211

[21] Benfatto G, Gruber Ch and Martin Ph-A 1983 Helv. Phys. Act. 5763

[22] Lighthill M J 1964 Introduction to Fourier analysis and generalised functions (Cambridge: University Press)

[23] Tu Y and Weichman P B 1994 Phys. Rev. Lett. 736

[24] Nieuwenhuizen Th M 1995 Phys. Rev. Lett. 744293

[25] Vojta T 1996 Phys. Rev. B $\mathbf{5 3} 710$ 